\title{
Redução nos níveis de ansiedade e depressão de pacientes com doença pulmonar obstrutiva crônica (DPOC) participantes de um programa de reabilitação pulmonar*
}

\author{
Dagoberto Vanoni de Godoy ${ }^{1}$, Rossane Frizzo de GodoY ${ }^{2}$
}

\begin{abstract}
Objetivos: A abordagem multidisciplinar oferecida por programas de reabilitação pulmonar (PRP) tem sido a melhor alternativa terapêutica para o tratamento de portadores de doença pulmonar obstrutiva crônica. Este ensaio clínico avaliou a prevalência de ansiedade e depressão e o efeito de um PRP sobre os níveis de ansiedade e depressão de 46 portadores de DPOC (idade: $62 \pm 11$ anos; 34 homens e 12 mulheres). Pacientes e métodos: Os pacientes participaram de um PRP com 12 semanas de duração: 24 sessões de exercícios físicos, 24 sessões de fisioterapia, 12 sessões de acompanhamento psicológico e três sessões educacionais. Todos os pacientes foram avaliados na linha de base e ao término do PRP através de três instrumentos: inventário de Beck para ansiedade (BAI); inventário de Beck para depressão (BDI), teste da caminhada de seis minutos (Tcam6'). Resultados: Os pacientes estudados demonstraram redução significativa nos níveis de ansiedade e depressão e melhora significativa no Tcam6': BAI 16,4 $\pm 6,9$ vs. $6,8 \pm 5,3$ ( $p<0,001$ ); BDI: $16,9 \pm 8,7$ vs. $7,5 \pm 6,6$

$(p<0,001)$; Tcam6': 335,7 \pm 83,4 vs. 441,6 \pm 100,8 ( $p<0,05)$. Conclusões: Pacientes com DPOC estudados apresentaram alta prevalência de ansiedade e depressão. O PRP foi capaz de reduzir de forma significativa os níveis de ansiedade e depressão, bem como de aumentar o desempenho do
\end{abstract} Tcam6'. (J Pneumol 2002;28(3):120-124)

\section{Reduction on the levels of anxiety and depression of COPD patients participating in a pulmonary rehabilitation program}

Study objectives: Multidisciplinary pulmonary rehabilitation has been the most suitable treatment for chronic obstructive pulmonary disease (COPD). This clinical trial studied the prevalence of anxiety and depression and the effect of a pulmonary rehabilitation program on anxiety and depression levels of 46 COPD patients (mean \pm SD age, $62 \pm 11$ years; 34 men and 12 women). Design: The participants underwent a 12-week treatment program: 24 sessions of physical exercise, 24 sessions of physiotherapy, 12 psychological sessions and three educational sessions. All patients were evaluated at baseline and at completion of the rehabilitation program through three instruments: Beck Anxiety Inventory (BAI), Beck Depression Inventory (BDI) and 6-

minute walking distance (Tcam6'). Results: Patients demonstrated significant statistical improvements, including reduced anxiety and depression, and increased endurance: BAI $16.4 \pm$ 6.9 vs. $6.8 \pm 5.3$ ( $p<0.001$ ); BDI: $16.9 \pm 8.7$ vs. $7.5 \pm 6.6$ ( $p<0.001$ ); Tcam6": $335.7 \pm 83.4$ vs. $441.6 \pm 100.8(p<0.05)$. Conclusions: COPD patients presented high prevalence of anxiety and depression levels. The pulmonary rehabilitation program was able to improve patient exercise performance, and to reduce anxiety and depression levels.

* Trabalho realizado no Ambulatório de Reabilitação Pulmonar, Serviço de Pneumologia e Cirurgia Torácica, Universidade de Caxias do Sul, Rio Grande do Sul, Brasil.

1. Mestre em Pneumologia - UFRGS; Professor de Pneumologia, Curso de Medicina, Centro das Ciências Biológicas e da Saúde, Universidade de Caxias do Sul.

2. Mestre em Ciências do Movimento Humano - UFRGS; Psicóloga do Ambulatório de Reabilitação Pulmonar da Universidade de Caxias do Sul.
Endereço para correspondência - Dagoberto Vanoni de Godoy, Av. Rio Branco, 209/103 - 95010-060 - Caxias do Sul, RS - Brasil. Tel. (54) 221-5006; e-mail: dvggodoy@ terra.com.br

Recebido para publicação em 3/9/01. Aprovado, após revisão, em 30/4/02. 
Descritores - DPOC. Reabilitação pulmonar. Execício físico. Ansiedade. Depressão.

Key words - COPD. Pulmonary rehabilitation. Physical exercise. Anxiety. Depression.

\section{INTRODUÇÃO}

A prevalência da doença pulmonar obstrutiva crônica tem aumentado progressivamente nas últimas décadas ${ }^{(1)}$. No Brasil, a Sociedade Brasileira de Pneumologia e Tisiologia estima a existência de 7,5 milhões de portadores de $D_{P O C}{ }^{(2)}$. Menezes et al. (1991) demonstraram prevalência de $12,7 \%$ de bronquite crônica na cidade de Pelotas (RS) ${ }^{(3)}$. Em termos mundiais, a Organização Mundial da Saúde (OMS) calculou prevalência de 9,3/1000 e 7,3/ 1000 habitantes, respectivamente, para homens e muIheres, em 1990(4).

Ansiedade, depressão e autoconceito depreciado podem estar presentes numa variedade de doenças agudas e crônicas ${ }^{(5)}$. Nas últimas duas décadas, um grupo expressivo de pesquisadores investigou as associações entre ansiedade, depressão e DPOC. Ansiedade tem sido identificada em pacientes com DPOC em taxas variando de $21 \%$ a $96 \%$ dos $\operatorname{casos}^{(6,7)}$. Depressão também é muito comum em DPOC, embora, algumas vezes, possa ser considerada desprovida de importância(8,9). Não obstante, distúrbios depressivos estão presentes em $27 \%$ a $79 \%$ desses indivíduos ${ }^{(10-12) .}$

Este estudo teve os seguintes objetivos: 1) avaliar a prevalência e a intensidade de ansiedade e depressão em um grupo de pacientes portadores de DPOC ao iniciarem um programa ambulatorial de reabilitação pulmonar (PRP); 2) ao término do PRP, avaliar o efeito deste sobre a intensidade de ansiedade e depressão desses mesmos indivíduos.

\section{Pacientes e mÉtOdos}

Este estudo foi realizado no Ambulatório de Reabilitação Pulmonar da Universidade de Caxias do Sul (UCS) entre outubro de 1999 e junho de 2001. Foram avaliados pacientes consecutivos portadores de DPOC, encaminhados ao PRP pelo Ambulatório da Disciplina de Pneumologia e Cirurgia Torácica da UCS. 0 protocolo de pesquisa foi aprovado pelo comitê de ética da instituição e todos os pacientes integrantes da pesquisa assinaram termo de consentimento informado.

\section{Seleção dos pacientes}

A avaliação médica constituiu-se na primeira etapa da seleção, visto que a confirmação do diagnóstico de DPOC foi condição sine qua non para a inclusão do paciente no estudo, constando dos seguintes itens: a) Anamnese e exame físico; b) Espirometria (Spirodoc, Medical Interna-
Siglas e abreviaturas utilizadas neste trabalho

BAI - Inventário de Beck para Ansiedade

BDI - Inventário de Beck para Depressão

C/I - Completo/Incompleto

CVF - Capacidade vital forçada

DPOC - Doença pulmonar obstrutiva crônica

Fem. - Feminino

OMS - Organização Mundial da Saúde

Masc. - Masculino

PRP - Programa de reabilitação pulmonar

RS - Rio Grande do Sul

Tcam6' - Teste da caminhada de seis minutos

$V E F_{1}$ - Volume expiratório forçado no 10 segundo

UCS - Universidade de Caxias do Sul

tional Research, Roma): a mensuração da função pulmonar foi realizada através de dois parâmetros espirométriCOS: volume expiratório forçado no primeiro segundo $\left(\mathrm{VEF}_{1}\right)$ ecapacidade vital forçada (CVF); c) Estudo radiológico convencional de tórax.

Foram incluídos adultos de ambos os sexos portadores de DPOC estadiados, segundo o I Consenso Brasileiro de DPOC, em três níveis de comprometimento: leve $\left(\mathrm{VEF}_{1} \geq\right.$ $60 \%$ do previsto), moderado $\left(\mathrm{VEF}_{1} \geq 40 \%<60 \%\right.$ do previsto) e grave $\left(\mathrm{VEF}_{1}<40 \% \text { do previsto }\right)^{(2)}$. Foram atribuídos os seguintes valores ao estadiamento: $D P O C$ leve $=1$; DPOC moderada $=2$; DPOC grave $=3$.

Os critérios de não inclusão foram os seguintes: a) incapacidade de cumprir o programa por dificuldades de deslocamento do domicílio até a sede do PRP e/ou indisponibilidade de tempo; b) presença de lesões músculoesqueléticas que contra-indicassem a realização de exercícios físicos; c) infecções respiratórias graves de repetição com necessidade de hospitalização.

Pacientes que utilizavam fármacos antidepressivos e ansiolíticos foram mantidos em tratamento com regime posológico inalterado. $\mathrm{Não}$ foram administrados fármacos antidepressivos ou ansiolíticos a pacientes que não faziam uso deles ao tempo do arrolamento para o estudo.

\section{Instrumentos de avaliação psicológica}

Dois instrumentos foram utilizados para a avaliação dos pacientes:

1) Níveis de ansiedade - Inventário Beck de Ansiedade (BAI). Esse inventário foi proposto por Beck para medir os sinto mas comuns de ansiedade ${ }^{(13)}$. 0 inventário consta de uma lista de 21 sintomas com quatro alternativas cada um, em ordem crescente do nível de ansiedade. Esse instrumento foi validado, no Brasil, por Cunha ${ }^{(14)}$. A classificação brasileira foi realizada com cinco mil casos, propondo os seguintes resultados: 0 a 9 - mínimo; 10 a 16 leve; 17 a 29 - moderado; e 30 a 63 - grave.

2) Níveis de depressão - Inventário de Beck para Depressão (BDI). Esse inventário foi proposto por Beck por 
constituir uma forma objetiva para medir as manifestações comportamentais da depressão. 0 inventário utilizado na pesquisa compreende 21 categorias de sintomas e atividades, com quatro alternativas cada um, em ordem crescente do nível de depressão. 0 paciente deveria escolher a que the parecesse mais apropriada(15). A soma dos escores identifica o nível de depressão. Esse instrumento foi validado por Cunha ${ }^{(14)}$. A classificação brasileira foi realizada com cinco mil casos, propondo o seguinte resultado: 0 a 11 - mínimo; 12 a 19 - leve; 20 a 35 moderado; e 36 a 63 - grave.

\section{Protocolo do programa de reabilitação pulmo-} nar

1) Duas sessões semanais de exercícios físicos, incluindo trabalho de membros superiores, membros inferiores e exercício aeróbico, através do uso de esteira ergométrica. O tempo de uso da esteira variou de acordo com as condições físicas de cada paciente. A intensidade do exercício foi limitada por sinais e sintomas. 0 objetivo principal foi exercitar o paciente em nível que variou de 75 a $85 \%$ de sua freqüência cardíaca máxima. Os exercícios eram suspensos se a freqüência cardíaca excedesse os limites acima apresentados, se houvesse arritmia cardíaca, pressão arterial acima de 180/110 mmH g, dor torácica, visão borrada, palidez, sudorese fria, dessaturação abaixo de $85 \%$ em paciente que já estivesse com oxigênio suplementar, e deterioração da coordenação motora e do nível de consciência.

2) Uma sessão de acompanhamento psicológico individual semanal, realizada por psicóloga.

3) Uma sessão de educação mensal grupal para discutir tópicos importantes sobre a DPOC.

4) Dois encontros semanais com a fisioterapeuta para trabalhar a reeducação respiratória.

Ao final das 12 semanas, os pacientes cumpriram um total de: 24 sessões de exercício, 24 sessões de reeducação respiratória, 12 sessões de acompanhamento psicológico individual e três sessões de educação.

Utilizou-se o desempenho no teste da caminhada de seis minutos (Tcam6') para avaliar a evolução na capacidade física dos sujeitos estudados.

\section{Análise estatística}

À análise estatística foi aplicado o princípio de intenção de tratar. Os dados quantitativos foram descritos através da média e desvio padrão. Nas variáveis categóricas utilizou-se o percentual. Os resultados foram analisados pelo teste $t$ de Student e teste do qui-quadrado. 0 nível de significância adotado foi de $\alpha=0,05$. Os dados foram processados e analisados com o auxílio dos programas SPSS for Windows v.6.0 e Epi-Info v.6.0.

\section{Resultados}

Entre outubro de 1999 e junho de 2001, 56 pacientes foram avaliados para a realização do PRP. Destes, 10 pacientes não foram incluídos: sete por impossibilidade de comparecer ao PRP devido à distância de sua moradia ou por indisponibilidade de tempo, dois por lesões em coluna vertebral secundárias à osteoporose e um por apresentar infecções respiratórias de repetição com necessidade de internações freqüentes.

Quarenta e seis pacientes realizaram o programa de reabilitação pulmonar. Os dados demográficos e de gravidade da doença estão demonstrados na Tabela 1. Três pacientes utilizavam antidepressivos inibidores da recaptação da serotonina.

Dos 46 pacientes, $73,9 \%$ eram homens e $26,1 \%$ muIheres. A média de idade da amostra foi de 63,28 anos; a maioria $(78,3 \%)$ apresentou nível de escolaridade até primeiro grau completo e estado civil de casado $(67,4 \%)$. A situação ocupacional indicou que 34 pacientes $(73,9 \%)$ não trabalhavam mais. A média de estadiamento da doença foi de 2,65 (doença moderada).

Nos procedimentos de avaliação inicial constatou-se que $67,6 \%$ dos homens e $75 \%$ das mulheres apresentavam ansiedade. A depressão foi diagnosticada em $58,8 \%$ da população masculina e $58,3 \%$ na feminina. Esses resultados podem ser verificados na Tabela 2 .

A pontuação média inicial do BAl foi de $16,46 \pm 6,9$, indicando ansiedade leve. No que se refere à pontuação média do BDI, o índice inicial foi $16,92 \pm 8,7$, caracteri-

TABELA 1

Dados demográficos e gravidade da DPOC

\begin{tabular}{lc}
\hline \multicolumn{1}{c}{ Características } & $\mathrm{N}=46$ \\
\hline Sexo, n (\%) & Masc. $=34(73,9)$ Fem. $=12(26,1)$ \\
\hline Idade (anos) & $63,28 \pm 11,4$ \\
\hline Escolaridade, $\mathrm{n}(\%)$ & $36(78,3)$ \\
$\quad$ Primária I/C & $7(15,2)$ \\
Secundária I/C & $3(6,5)$ \\
Superior I/C & $12(26,1)$ \\
\hline Situação ocupacional, n (\%) & $34(73,9)$ \\
$\quad$ Trabalha & \\
Não trabalha & $4(8,7)$ \\
\hline Estado civil, n (\%) & $31(67,4)$ \\
Solteiro & $4(8,7)$ \\
Casado & $7(15,7)$ \\
Separado & $2,65 \pm 0,56$ \\
Viúvo &
\end{tabular}


zando depressão leve. Após o cumprimento do programa de reabilitação pulmonar, houve redução das pontuações em ambos os instrumentos: BAI: $6,83 \pm 5,35$ ( $p<$ $0,001) ;$ BDI: $7,56 \pm 6,6(p<0,001)$. Esses resultados indicam níveis mínimos de ansiedade e depressão, conforme demonstrado na Tabela 3. Pacientes com estágio de DPOC 2 apresentaram-se mais ansiosos e mais deprimidos em relação aos indivíduos com estágio 3: BAI inicial $=18,66 \pm 18$ versus $11,16 \pm 7(p=0,4)$ e BDI inicial $=20,6 \pm 17$ versus $13,97 \pm 9(p=0,5)$, mas não diferiram estatisticamente.

Ainda na Tabela 3, encontram-se expressas as médias dos resultados do Tcam6' inicial e final.

TABELA 2

Prevalência de ansiedade e depressão segundo o sexo aferidas pelo BAI e BDI

\begin{tabular}{cccc}
\hline Instrumento & Masculino (\%) & Feminino $(\%)$ & Total $(\%)$ \\
\hline BAI & $23(67,8)$ & $9(75)$ & $32(69,5)$ \\
BDI & $20(58,8)$ & $7(58,3)$ & $27(58,6)$ \\
\hline
\end{tabular}

TABELA 3

Evolução dos níveis de ansiedade, depressão e da distância caminhada em seis minutos

\begin{tabular}{lccc}
\hline Instrumento & Inicial & Final & Valor de $p$ \\
\hline BAI & $16,46 \pm 6,9$ & $6,83 \pm 5,3$ & $<0,001$ \\
BDI & $16,92 \pm 8,7$ & $7,56 \pm 6,6$ & $<0,001$ \\
Tcam6' & $335,7 \pm 83,4$ & $441,6 \pm 100,8$ & $<0,005$ \\
\hline
\end{tabular}

\section{DISCUSSÃo}

O impacto da DPOC sobre o indivíduo portador não se dá somente na limitação física para a execução das atividades da vida diária, mas, também, nas relações afetivas, conjugais e sexuais, no lazer e no exercício profissional|(8,16). Em decorrência dessa situação, muitos pacientes tornamse amplamente dependentes de seus familiares, o que acaba reforçando seu sentimento de incapacidade e contribuindo para a diminuição de sua auto-estima (17-19).

No presente estudo, a maior parte da amostra foi composta por homens com DPOC de moderada a grave, na faixa de 60 anos de idade, casados, com baixo nível de escolaridade, e que não trabalhavam, não diferindo das populações analisadas em outros estudos de programas de reabilitação pulmonar ${ }^{(8)}$.

Ansiedade e a depressão são comuns em pacientes portadores de $D P O C^{(20-24)}$. Foram utilizados do is instrumentos de mensuração para aferir objetivamente as modifica- ções comportamentais relacionadas à ansiedade (BAI) e à depressão (BDI). Os referidos instrumentos, entretanto, não são capazes de firmar, isoladamente, o diagnóstico de depressão e/ou ansiedade. Porém, nossos diagnósticos foram confirmados através de entrevistas com psicóloga. Dos componentes da amostra, $67,6 \%$ dos homens e $75 \%$ das mulheres apresentaram ansiedade. White et al., em seus achados, identificaram $40 \%$ de ansiedade em pacientes portadores de DPOC ${ }^{(25)}$. Agle e Baum constataram níveis de ansiedade em mais de $96 \%$ dos casos dos pacientes com DPOC ${ }^{(26)}$.

$\mathrm{Na}$ linha de base, a depressão estava presente em $58,8 \%$ da população masculina e em $58,3 \%$ da feminina. Emery et al. verificaram depressão em taxas de $51 \%$ a $74 \%$ dos $\operatorname{casos}^{(12)}$. Kaplan et al. demonstraram prevalência de $42 \%$ de depressão moderada ou grave ${ }^{(11)}$. Numa pesquisa realizada com pacientes com DPOC na rede hospitalar de Porto Alegre, depressão maior foi encontrada em $26,9 \%$ dos pacientes ${ }^{(10)}$.

A alta prevalência de ansiedade e depressão nos pacientes com DPOC pode estar associada a diferentes motivos. A ansiedade relaciona-se principalmente à intensa dispnéia, queixa mais comum dos pacientes que sofrem dessa doença(28). Em função do temor da dispnéia, evitam o esforço físico. Isso acentua seu descondicionamento físico, estabelecendo-se um ciclo vicioso de agravamento constante da dispnéia ${ }^{(23,29)}$. Em relação à depressão, uma possível explicação é de que esta seria uma resposta psicológica do paciente à medida que este se confronta com as significantes limitações para realizar as atividades da vida diária e com o esforço exigido para ajustar-se à incapacidade. Com a deterioração gradual imposta pela doença, o paciente apresenta "perdas" em diversas áreas: lazer, social, profissional, sexual, interpessoal(16,19,30,31). Ressalte-se que nossos resultados não puderam ser comparados com outros estudos da literatura porque estes não utilizaram BAI e BDI na sua metodologia.

Ao final do PRP, os pacientes do grupo estudado meIhoraram sua capacidade de realização de exercícios e reduziram seus níveis de ansiedade e depressão de maneira significativa, conforme os resultados demonstrados pelos Tcam6', BAI e BDI, respectivamente. A melhora observada provavelmente deva-se ao somatório de pequenos ganhos nos seguintes aspectos: capacidade aeróbica, motivação, dessensibilização da dispnéia, treinamento da musculatura respiratória e aprimoramento das técnicas de desempenho das atividades da vida diária.

Em conclusão, este estudo confirma os achados prévios de altos índices de ansiedade e depressão em pacientes com DPOC. Embora a ansiedade tenha aparecido em ambos os sexos, foi maior no feminino. Os índices de depressão foram semelhantes. Nossos resultados ratificam a capacidade doS PRP em melhorar o desempenho 
físico e reduzir os níveis de ansiedade e depressão em pacientes com DPOC. O manejo interdisciplinar e individualizado propiciado a paciente com DPOC mostrou-se

\section{REFERÊNCIAS}

1. Knorst M, Menezes AMB. Epidemiologia da doença pulmonar obstrutiva crônica. In: Corrêa da Silva LC, Menezes AMB. Epidemiologia das Doenças Respiratórias. Rio de J aneiro: Revinter, 2001;57-75.

2. Sociedade Brasileira de Pneumologia e Tisiologia. I Consenso Brasileiro de Doença Pulmonar Obstrutiva Crônica (DPOC). J Pneumol 2000; 26:S1-S52.

3. Menezes AMB, Victora CG, Rigatto M. Prevalence and risk factors for chronic bronchitis in Pelotas, RS, Brazil: a population-based study. Thorax 1994;49:1217-21.

4. Murray CLJ, Lopez AD. Evidence-based health policy-lessons from the global burden of disease study. Science 1996;274:740-3.

5. Hetem $L A B$. Diagnóstico diferencial dos transtornos de ansiedade. In: Hetem LAB, Graff FG. Ansiedade e transtornos de ansiedade. Rio de Janeiro: ECN, 1997;228-47.

6. Janssens J P, Rochat T, Frey J G. Health-related quality of life in patients under long-term oxygen therapy: a home-based descriptive study. Respir Med 1997;91:592-602.

7. White RJ, Rudkin ST, Ashley J. Outpatient pulmonary rehabilitation in severe chronic obstructive pulmonary disease. J R Coll Physicians Lond 1997;31:541-5.

8. ACCP/AACVPR. Pulmonary Rehabilitation Guidelines Panel. ACCP/ AACVPR evidence based guidelines. Chest 1997;112:1363-96.

9. Mahler D. Pulmonary rehabilitation. Chest 1998;113:2635-89.

10. Heckler $M$, Weingartner $R$, Moreira J S. Prevalência de depressão maior em pacientes com doença pulmonar obstrutiva crônica. J Pneumol 1997;23:231-6.

11. Kaplan R, Eakin E, Ries AL. Psychosocial issues in the rehabilitation of patients with chronic obstructive pulmonary disease. In: Casabury $R$, Petty $T L$, editors. Principles and practice of pulmonary rehabilitation. Philadelphia: WB Saunders, 1993;351-65.

12. Emery CF, Leatherman NE, Burker EJ, Maclntyre NR. Psychological outcomes of a pulmonary rehabilitation program. Chest 1991;100:613-7.

13. Cunha J A. Generalidades sobre a versão brasileira do BDI, BAI, BHS e BSI. Anais do $7^{\circ}$ Encontro Nacional sobre Testes Psicológicos. $1^{0}$ Congresso Ibero-Americano de Avaliação Psicológica. Porto Alegre, 1997;135-9.

14. Cunha JA. Estudos dos pontos de corte do BDI e BAI na versão em português. 8o Congresso Nacional de Avaliação Psicológica. Poster 78, Porto Alegre, 1999.

15. Cunha JA. Psicodiagnóstico-R. Porto Alegre: Artes Médicas, 1993.

16. Selecky P. Sexuality and the patient with lung disease. In: Casabury $R$, Petty TL. Principles and practice of pulmonary rehabilitation. Philadelphia: WB Saunders, 1993;382-91.

17. Dudley D, Sitzman J. Psychobiologic evaluation and rehabilitation in pulmonary disease. In: Casabury R, Petty TL. Principles and practice benéfico no período de observação. Contudo, nossos dados não permitem estimar se a melhora será duradoura após o término do PRP.

of pulmonary rehabilitation. Philadelphia: WB Saunders, 1993; 25273.

18. Inniss P. Psychosocial aspects of pulmonary rehabilitation. In: Haas F, Axen K. Pulmonary therapy and rehabilitation: principles and practice. $2^{\text {nd }}$ ed., Baltimore: Williams \& Wilkins, 1991;289-99.

19. Lustig F. Vocational rehabilitation. In: Haas F, Axen K. Pulmonary therapy and rehabilitation: principles and practice. $2^{\text {nd }}$ ed. Baltimore: Williams \& Wilkins, 1991;300-14

20. Emery C, Hauck ER, Schein RL, Maclntyre NR. Psychological and cognitive outcomes of a randomized trial of exercise among patients with chronic obstructive pulmonary disease. Health Psychol 1998;17: 232-40.

21. Gayle R, Spitler DL, Karper WB, lager RM, Rice SN. Psychological changes in exercising COPD patients. Int J Rehabil Res1988;11:33542.

22. Niederman M. Introduction: mechanisms and management of COPD. We can do better. It's time for a reevaluation. Chest 1998;113:2335S$45 \mathrm{~S}$.

23. Leatherman N. Pulmonary rehabilitation. In: Dantzker D, Maclntyre NR, Bakow ED. Comprehensive respiratory care. Philadelphia: WB Saunders, 1995;925-48.

24. Smoller JW, Pollack MH, Otto MW, Rosenbaum IF, Kradin RL. Panic, anxiety, dyspnea and respiratory disease. Theoretical and clinical considerations. Am J Respir Crit Care Med 1996;154:6-17.

25. White RJ, Rudkin ST, Ashley I, Stevens VA, Burrows S. Outpatient pulmonary rehabilitation in severe chronic obstructive pulmonary disease. J R Coll Physicians Lond 1997;31:541-5.

26. Agle DP, Baum GL. Psychosocial aspects of chronic obstructive pulmonary disease. Med Clin North Am 1977;61:749-58.

27. Carrieri-Kholman K, Gormley IM, Douglas MK, Paul SM, Stulbarg MS. Exercise training decreases dyspnea and distress and anxiety associated with it. Chest 1996;110:1526-35.

28. Hodgkin JE. Chronic obstructive pulmonary disease. Clin Chest Med 1990;11:363-9.

29. Hodgkin J E. Pulmonary rehabilitation. In: Hodgkin J E, Petty TL. Chronic obstructive pulmonary disease: current concepts. Philadelphia: W.B. Saunders, 1987; 154-71.

30. Constain JS, Hass S, Schicc J S. Sexual aspects of the pulmonaryimpaired person. In: Haas F, Axen K. Pulmonary therapy and rehabilitation: principles and practice. $2^{\text {nd }}$ ed. Baltimore: Williams $\&$ Wilkins, 1991;315-26.

31. Jones $N$, Berman W, Bartkiewicz P, Oldridge N. Distúrbios respiratórios obstrutivos crônicos. In: Skinner, J ones S. Prova de esforço. A prescrição de exercícios para casos específicos. Rio de Janeiro: Ed. Revinter, 1991;191-204. 